EPJ manuscript No.

(will be inserted by the editor)

\title{
Fluid transport at low Reynolds number with magnetically actuated artificial cilia
}

\author{
Erik M. Gauger ${ }^{1}$, Matthew T. Downton ${ }^{2}$, and Holger Stark ${ }^{2}$ \\ 1 Department of Materials, University of Oxford, Parks Road, Oxford, OX1 3PH, UK \\ 2 Institut für Theoretische Physik, Technische Universität Berlin, Hardenbergstr. 36, D-10623 Berlin, Germany
}

November 1, 2018

\begin{abstract}
By numerical modeling we investigate fluid transport in low-Reynolds-number flow achieved with a special elastic filament or artifical cilium attached to a planar surface. The filament is made of superparamagnetic particles linked together by DNA double strands. An external magnetic field induces dipolar interactions between the beads of the filament which provides a convenient way of actuating the cilium in a well-controlled manner. The filament has recently been used to successfully construct the first artificial micro-swimmer [R. Dreyfus at al., Nature 437, 862 (2005)]. In our numerical study we introduce a measure, which we call pumping performance, to quantify the fluid transport induced by the magnetically actuated cilium and identify an optimum stroke pattern of the filament. It consists of a slow transport stroke and a fast recovery stroke. Our detailed parameter study also reveals that for sufficiently large magnetic fields the artificial cilium is mainly governed by the Mason number that compares frictional to magnetic forces. Initial studies on multi-cilia systems show that the pumping performance is very sensitive to the imposed phase lag between neighboring cilia, i.e., to the details of the initiated metachronal wave.
\end{abstract}

PACS. 87.19.St - 87.16.Ac - 87.16.Qp

\section{Introduction}

Fluid transport and mixing on the microscopic level at low Reynolds numbers is a fascinating problem that is at the center of a successful lab-on-chip technology [1. Nature has provided an ingenious solution to this challenge by using long elastic filaments, called flagella or cilia, that are actuated internally by molecular motors $2,3,4$. The resulting beating patterns, in general three-dimensional, ' have to be non-reciprocal as Purcell has taught us [5]. Nature uses arrays of collectively beating cilia to transport mucus in the respiratory tract, fluid in the brain [6], or to propel microorganisms such as the paramecium. During an early stage of a developing embryo, arrays of rotating cilia are responsible for establishing the left-right asymmetry in the placement of organs [7. Recently, experimental efforts have been initiated to copy nature's successful concept by developing biomimetic or artificial cilia that are actuated by external fields [1,8,9 or to move fluid with the help of bacterial carpets 10. On the other hand, there has been an increasing interest in recent times in contributing to the theoretical understanding of how single cilia or flagella function (e.g. Refs. 11, 12, 13, 14, 15, 16, [17, 18, 19]) and of how their collective beating patterns, known as metachronal waves, occur (e.g. Refs. 12,17,20, 21,22, 23, 24, 25, 26]).

Some years ago, Dreyfus et al. introduced the first artificial micro-swimmer 8 based on an artificial flagel- lum. This elastic filament consists of superparamagnetic micron-sized beads that are linked together by pieces of double-stranded DNA 27,28. The flagellum is actuated by an oscillating magnetic field so that it drags an attached red-blood cell forward. Modeling using a continuum [15] or a discretized [18] approach described the behavior of the swimmer very well.

Based on our theoretical work in Ref. [18, we explore in this article by numerical modeling how the artificial flagellum or cilium can be employed for fluid transport. We attach the cilium to a surface and actuate it by an external magnetic field, the direction of which oscillates about the surface normal in an asymmetric fashion. To achieve fluid transport, the stroke pattern has to be asymmetric [17. We therefore introduce a slow transport stroke, where the cilium remains nearly straight, and a fast recovery stroke, where the cilium bends due to increased hydrodynamic friction. A measure for the amount of transported fluid during one beating cycle, called pumping performance, helps us to discuss the system in detail and to identify the optimum conditions under which the cilium should be operated. We also look at multi-cilia systems with a defined phase lag between neighboring cilia and illustrate that such a phase lag is advantageous for fluid transport. Of course, our metachronal waves are controlled by the external field and do not occur through self-organized synchronization of the beating cilia. There is now strong evidence that this intriguing feature of biological cilia ar- 


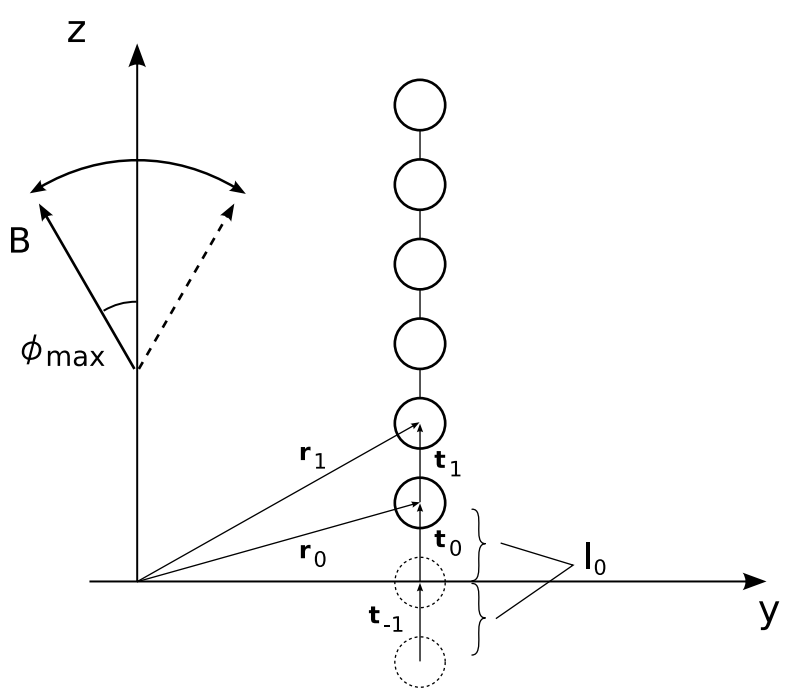

Fig. 1. Filament attached perpendicular to a bounding wall. The first three bonds $\boldsymbol{t}_{-1}, \boldsymbol{t}_{0}$ and $\boldsymbol{t}_{1}$ are shown. The actual filament starts with the bead at $\boldsymbol{r}_{0}$, whereas the virtual beads drawn as dotted lines are kept at fixed positions to anchor the filament perpendicular to the $x y$ plane through their elastic force contributions. The actuating magnetic field oscillating around the $z$ axis is also sketched.

rays is caused by hydrodynamic interactions $12,17,20,21$, 22, 23, 24, 25, 26]). Similar synchronization phenomena mediated by hydrodynamic interactions were discussed for sperm cells by Taylor [30] and for helical flagella in Ref. 31 .

The article is organized as follows. Section 2 explains our modeling of the artificial cilium including the treatment of hydrodynamic interactions close to surfaces and the magnetic actuation cycle of the filament. Section 3 introduces the pumping performance as a measure for the transported fluid. In Sections 4 and [5 we then discuss the pumping performance for a single cilium and for multicilia systems. The article ends with a conclusion.

\section{Model}

The superparamagnetic filament is modeled by a beadspring configuration, which additionally resists bending like a worm-like chain 32 . Consequently, each bead of the filament is subject to stretching and bending forces, for which the chemical linkers are responsible, and to dipolar interaction forces due to the induced magnetic dipoles of the beads. We completely ignore the contributions of the chemical linkers to hydrodynamic friction, so the filament interacts with the fluid surrounding solely via the hydrodynamic friction of the beads. As illustrated in Fig. 11 the filament is attached to a planar surface with the help of two virtual beads that fix its position and give it an orientation orthogonal to the surface. These virtual beads contribute to elastic forces but do not participate in hydrodynamic or dipolar interactions.

In the following, we will summarize the forces, acting on each bead within the filament, and the equations of motion. Details of the derivations are given in Ref. [18. The equations of motion contain hydrodynamic mobilities which we construct up to Rotne-Prager level based on the appropriate Green function commonly called Blake's tensor 33, 34. This guarantees a vanishing fluid velocity field at the bounding surface. The description of the time protocol of the actuating magnetic field concludes the section.

\subsection{Energies and forces of the superparamagnetic filament}

The total free energy for our bead-spring model is given by a sum over dipolar and elastic-energy contributions [18],

$$
H=H^{D}+H^{S}+H^{B},
$$

where $H^{D}$ is the dipole-dipole interaction energy, $H^{S}$ is the stretching energy obeying Hooke's law and $H^{B}$ is the discretized free bending energy of an elastic rod. As in our previous work, the actuation and hence also the motion of the filament takes place in the $y z$ plane and no twisting of the filament occurs, allowing us to ignore a twisting energy in our model. We shall briefly discuss the individual energy contributions of Eq. (1) in the following.

Let $\boldsymbol{t}_{i}$ be the vector connecting the centers of the two adjacent beads labeled $i$ and $i-1$. A deviation $l_{i}=\left|\boldsymbol{t}_{i}\right|$ of the beads from their equilibrium spacing $l_{0}$ then gives a total stretching free energy of

$$
H^{S}=\frac{1}{2} k \sum_{i=0}^{N-1}\left(l_{i}-l_{0}\right)^{2},
$$

where $k$ is the stretching constant and $N$ the total number of beads.

Discretizing the continuum bending energy of the wormlike chain model [32,35, we obtain by a straightforward calculation 18

$$
H^{B}=\frac{A}{l_{0}} \sum_{i=-1}^{N-2}\left(1-\hat{\boldsymbol{t}}_{i+1} \cdot \hat{\boldsymbol{t}}_{i}\right),
$$

where $\hat{\boldsymbol{t}}_{i}=\boldsymbol{t}_{i} / l_{i}$ and $A$ is the bending stiffness.

Finally, consider two identical paramagnetic beads with radius $a$ and magnetic susceptibility $\chi$ subject to a homogeneous external magnetic field $\boldsymbol{B}$. Both beads develop a dipole moment with identical orientation and strength,

$$
\boldsymbol{p}=\frac{4 \pi a^{3}}{3 \mu_{0}} \chi \boldsymbol{B}
$$

where $\mu_{0}=4 \pi \times 10^{-7} \mathrm{~N} / \mathrm{A}^{2}$ is the permeability of free space. The resulting dipoles of the beads of the filament give rise to the total dipole-dipole interaction energy

$$
H^{D}=\frac{4 \pi a^{6}}{9 \mu_{0}}(\chi B)^{2} \sum_{i<j}^{N-1} \frac{1-3\left(\hat{\boldsymbol{p}} \cdot \hat{\boldsymbol{r}}_{i j}\right)}{r_{i j}^{3}}
$$


where $r_{i j}=\left|\boldsymbol{r}_{j}-\boldsymbol{r}_{i}\right|, \hat{\boldsymbol{r}}_{i j}=\left(\boldsymbol{r}_{j}-\boldsymbol{r}_{i}\right) / r_{i j}$ and the double sum runs over all terms with $i<j$, where the minimal $i$ is 0 and the maximal $j$ is $N-1$.

Now that all relevant interaction energies have been characterized, the non-hydrodynamic force acting on each bead is obtained as usual,

$$
\boldsymbol{F}_{i}=-\boldsymbol{\nabla}_{\boldsymbol{r}_{i}}\left(H^{S}+H^{B}+H^{D}\right),
$$

where $\boldsymbol{\nabla}_{\boldsymbol{r}_{i}}$ is the gradient operator with respect to $\boldsymbol{r}_{i}$. The total non-hydrodynamic force $\boldsymbol{F}_{i}=\boldsymbol{F}_{j}^{S}+\boldsymbol{F}_{j}^{B}+\boldsymbol{F}_{j}^{D}$ acting on bead $i$ is readily calculated from Eqs. (2) 3, 5). Explicit expressions of $\boldsymbol{F}_{i}$ can also be found in Ref. [18.

\subsection{Equations of Motion}

On the micron length scale, the motion of a particle immersed in a viscous fluid, such as water, is entirely dominated by friction and the particle's inertia can be neglected provided the time scale of interest exceeds the momentum relaxation time [36]. Hence, the velocities $\boldsymbol{v}_{i}$ of the beads are proportional to the forces $\boldsymbol{F}_{j}$ acting on them and the beads obey the following equations of motion 36]

$$
\boldsymbol{v}_{i}=\sum_{j} \boldsymbol{\mu}_{i j} \boldsymbol{F}_{j} \text { with } \boldsymbol{F}_{j}=\boldsymbol{F}_{j}^{S}+\boldsymbol{F}_{j}^{B}+\boldsymbol{F}_{j}^{D} .
$$

All the forces depend on the spatial configuration of the filament, i. e., the beads' locations $\boldsymbol{r}_{i}$. Furthermore, the dipolar forces also possess an explicit time dependence through the external magnetic field, which we use to actuate the filament.

Hydrodynamic friction enters the equations of motion via the mobilities $\boldsymbol{\mu}_{i j}$, which depend on the geometrical configuration of the beads. The flow field induced by one moving bead creates a drag on all other beads in the vicinity and thus indirectly displaces them. Since induced flow fields are long ranged (they decay as $1 / r$, where $r$ is the distance from a moving bead), hydrodynamic interactions play an important role in viscous systems with low Reynolds number. While hydrodynamic interactions constitute a highly complicated many-body problem [36, their leading order is given by two-particle interactions. The relatively simple Rotne-Prager approximation can be employed whenever the beads are not so close together that lubrication effects need to be considered [36,37. However, the surface, to which our filament is attached, introduces significantly more complexity due to the no-slip boundary condition even when we restrict ourselves to pairwise interactions at the Rotne-Prager level.

\subsection{Hydrodynamic interactions}

In the low Reynolds number regime, the fluid flow velocity at an arbitrary point $\boldsymbol{r}$ is linearly related to a point force $\boldsymbol{F}_{0}$ at $\boldsymbol{r}^{\prime}$ by 36]

$$
\boldsymbol{u}(\boldsymbol{r})=\frac{\boldsymbol{G}\left(\boldsymbol{r}-\boldsymbol{r}^{\prime}\right)}{8 \pi \eta} \boldsymbol{F}_{0}
$$

where $\boldsymbol{u}(\boldsymbol{r})$ denotes the flow field at $\boldsymbol{r}$ and $\eta$ is the fluid's viscosity. In an unbounded fluid, the Green function $\boldsymbol{G}(\boldsymbol{r}-$ $\boldsymbol{r}^{\prime}$ ), commonly referred to as Oseen tensor in literature, is given by

$$
\boldsymbol{G}(\boldsymbol{r})=\frac{1}{r} \boldsymbol{I}+\frac{\boldsymbol{r} \otimes \boldsymbol{r}}{r^{3}} .
$$

Here, $\boldsymbol{I}$ is the $3 \times 3$ identity matrix and $\otimes$ denotes the dyadic product.

The aforementioned Rotne-Prager mobilities of spherical particles are commonly used quantities in an unbounded fluid, which can be derived from the Oseen tensor 36. The respective self and cross mobilities are given by the following expressions for two spheres of radius $a$,

$$
\begin{aligned}
\boldsymbol{\mu}_{i i}= & \mu_{0} \boldsymbol{I}, \\
\boldsymbol{\mu}_{i j}^{r p}= & \mu_{0}\left\{\frac{3}{4} \frac{a}{r_{i j}}\left[\boldsymbol{I}+\hat{\boldsymbol{r}}_{i j} \otimes \hat{\boldsymbol{r}}_{i j}\right]\right. \\
& \left.+\frac{1}{2}\left(\frac{a}{r_{i j}}\right)^{3}\left[\boldsymbol{I}-3 \hat{\boldsymbol{r}}_{i j} \otimes \hat{\boldsymbol{r}}_{i j}\right]\right\}, i \neq j,
\end{aligned}
$$

where $\boldsymbol{r}_{i j}=\boldsymbol{r}_{i}-\boldsymbol{r}_{j}$ and $\hat{\boldsymbol{r}}_{i j}=\boldsymbol{r}_{i j} / r_{i j}$ and $\mu_{0}=(6 \pi \eta a)^{-1}$.

However, in close proximity to a planar surface with no-slip boundary condition, the traditional Rotne-Prager mobilities can no longer be employed. The pressure and velocity fields of a point force for this boundary condition have been known for a long time and were first derived by Lorentz [40. Blake put these results into a modern form replacing the Oseen tensor by the appropriate Green function [33. The condition of a vanishing fluid velocity field on an infinitely extended plane is satisfied with the help of appropriate mirror images, similar to the image charge approach often used in electrostatics. In contrast to electrostatics, where it suffices to simply mirror the charge distribution, the hydrodynamic image system is more involved due to the more complicated structure of the Stokes equation when compared to the Poisson equation. Hence, socalled stresslet and source-dipole contributions are needed in addition to the stokeslet of the point force and its mirrored point disturbance (also called anti-stokeslet). This yields Blake's tensor,

$$
\begin{aligned}
\boldsymbol{G}^{\text {Blake }}\left(\boldsymbol{r}, \boldsymbol{r}^{\prime}\right)= & \boldsymbol{G}\left(\boldsymbol{r}-\boldsymbol{r}^{\prime}\right)+\boldsymbol{G}^{i m}\left(\boldsymbol{r}, \overline{\boldsymbol{r}}^{\prime}\right) \\
= & \boldsymbol{G}\left(\boldsymbol{r}-\boldsymbol{r}^{\prime}\right) \\
& -\boldsymbol{G}\left(\boldsymbol{r}-\overline{\boldsymbol{r}}^{\prime}\right)+\delta \boldsymbol{G}^{i m}\left(\boldsymbol{r}, \overline{\boldsymbol{r}}^{\prime}\right),
\end{aligned}
$$

where $\boldsymbol{G}\left(\boldsymbol{r}-\boldsymbol{r}^{\prime}\right)$ and $\boldsymbol{G}\left(\boldsymbol{r}-\overline{\boldsymbol{r}}^{\prime}\right)$ are Oseen tensors, $\boldsymbol{r}^{\prime}$ is the coordinate vector of the stokeslet source, and $\overline{\boldsymbol{r}}^{\prime}$ is the position of the anti-stokeslet source, i. e., the stokeslet source mirrored at the bounding $x y$ plane $\left[\boldsymbol{r}=(x, y, z), \overline{\boldsymbol{r}}^{\prime}=\right.$ $\left.\left(x^{\prime}, y^{\prime},-z^{\prime}\right)\right]$. Finally, $\delta \boldsymbol{G}^{i m}\left(\boldsymbol{r}, \overline{\boldsymbol{r}}^{\prime}\right)$ denotes the source-dipole and stresslet contributions.

For particles that are far apart from each other, it suffices to use the Blake tensor for the mobility functions, i. e., to treat the particles as point-like objects, as is frequently done 23, 41. However, if the particles approach each other, their finite sizes become relevant as is the case in our filament. To take this effect into account, we derive the mobility functions in Eq. (7) up to the Rotne-Prager 
level following Refs. 42,43. Consider $\boldsymbol{f}\left(\boldsymbol{r}^{\prime}\right)$ to be the force density on the surface $\partial V_{j}$ of a sphere $j$ with its centre at $\boldsymbol{r}_{j}$. The force density acts on the fluid and induces the flow field

$$
\boldsymbol{u}(\boldsymbol{r})=\frac{1}{8 \pi \eta} \int_{\partial V_{j}} \boldsymbol{G}^{\text {Blake }}\left(\boldsymbol{r}, \boldsymbol{r}^{\prime}\right) \boldsymbol{f}\left(\boldsymbol{r}^{\prime}\right) d S^{\prime}
$$

The leading order of $\boldsymbol{f}\left(\boldsymbol{r}^{\prime}\right)$ is $\boldsymbol{F}_{j} /\left(4 \pi a^{2}\right)$, where $\boldsymbol{F}_{j}$ denotes the total force on particle $j$. Taking it into account and expanding $\boldsymbol{G}^{\text {Blake }}\left(\boldsymbol{r}, \boldsymbol{r}^{\prime}\right)$ about $\boldsymbol{r}^{\prime}=\boldsymbol{r}_{j}$ yields for the flow field

$$
\boldsymbol{u}(\boldsymbol{r}) \approx \frac{1}{8 \pi \eta}\left[\left(1+\frac{a^{2}}{6} \nabla_{\boldsymbol{r}^{\prime}}^{2}\right) \boldsymbol{G}^{\text {Blake }}\left(\boldsymbol{r}, \boldsymbol{r}^{\prime}\right)\right]_{\boldsymbol{r}^{\prime}=\boldsymbol{r}_{j}} \boldsymbol{F}_{j}
$$

We proceed by calculating the effect of $\boldsymbol{u}(\boldsymbol{r})$ on the motion of another sphere $i$ with the help of Faxén's theorem [36],

$$
\boldsymbol{v}_{i}=\frac{1}{6 \pi \eta a} \boldsymbol{F}_{i}+\left(1+\frac{1}{6} a^{2} \nabla_{\boldsymbol{r}_{i}}^{2}\right) \boldsymbol{u}\left(\boldsymbol{r}_{i}\right) .
$$

Note that $\boldsymbol{u}\left(\boldsymbol{r}_{i}\right)$ also includes the flow fields created by the mirror image of sphere $i$, i.e., by the term $\boldsymbol{G}^{i m}\left(\boldsymbol{r}, \overline{\boldsymbol{r}}^{\prime}\right)$ in the Blake tensor of Eq. (12). Combining Eqs. (14) and (15), we immediately obtain the cross mobilities [44]

$\boldsymbol{\mu}_{i j}=\frac{1}{8 \pi \eta}\left[\left(1+\frac{a^{2}}{6} \boldsymbol{\nabla}_{\boldsymbol{r}_{i}}^{2}\right)\left(1+\frac{a^{2}}{6} \boldsymbol{\nabla}_{\boldsymbol{r}_{j}}^{2}\right) \boldsymbol{G}^{\text {Blake }}\left(\boldsymbol{r}_{i}, \boldsymbol{r}_{j}\right)\right]$,

and the self mobility of particle $i$,

$$
\begin{aligned}
\boldsymbol{\mu}_{i i}= & \frac{1}{8 \pi \eta}\left[\frac{4}{3 a} \boldsymbol{I}+\left(1+\frac{a^{2}}{6} \nabla_{\boldsymbol{r}_{i}}^{2}\right)\right. \\
& \left.\left(1+\frac{a^{2}}{6} \boldsymbol{\nabla}_{\overline{\boldsymbol{r}}_{i}}^{2}\right) \boldsymbol{G}^{i m}\left(\boldsymbol{r}_{i}, \overline{\boldsymbol{r}}_{i}\right)\right] .
\end{aligned}
$$

Application of the differential operators in Eqs. (16) and (17) to the Oseen tensors $\boldsymbol{G}\left(\boldsymbol{r}-\boldsymbol{r}^{\prime}\right)$ and $\boldsymbol{G}\left(\boldsymbol{r}-\overline{\boldsymbol{r}}^{\prime}\right)$ in the Blake tensor simply gives Rotne-Prager matrices. Hence, we write the mobilities as

$$
\begin{aligned}
& \boldsymbol{\mu}_{i i}=\mu_{0} \boldsymbol{I}-\boldsymbol{\mu}^{r p}\left(\boldsymbol{r}_{i}-\overline{\boldsymbol{r}}_{i}\right)+\delta \boldsymbol{\mu}_{\text {self }}, \\
& \boldsymbol{\mu}_{i j}=\boldsymbol{\mu}^{r p}\left(\boldsymbol{r}_{i}-\boldsymbol{r}_{j}\right)-\boldsymbol{\mu}^{r p}\left(\boldsymbol{r}_{i}-\overline{\boldsymbol{r}}_{j}\right)+\delta \boldsymbol{\mu}_{i j},
\end{aligned}
$$

where $\boldsymbol{\mu}^{r p}(\boldsymbol{r})$ are the Rotne-Prager tensors defined by Eq. (11) and $\delta \boldsymbol{\mu}_{\text {self }}$ and $\delta \boldsymbol{\mu}_{i j}$ are the sourcelet and stresslet contributions originating from $\delta \boldsymbol{G}^{i m}$. These evaluate to more complicated expressions which we give in Appendix B.

\subsection{Reduced Equations of Motion}

We have shown in previous work that the number of system parameters for the superparamagnetic filament can be significantly reduced by introducing just three dimensionless variables [18. The essential parameters governing the dynamics of the filament were identified by rescaling the dynamic equations appropriately so that only reduced variables appeared. Instead of repeating the derivation of these reduced variables given in Ref. [18, we limit ourselves to discussing their physical significance.

One of the emerging quantities is

$$
S_{p}=\left(\frac{6 \pi \eta \frac{a}{l_{0}} \omega L^{4}}{A}\right)^{1 / 4}=\frac{L}{l_{h}},
$$

which we call 'sperm number' in the following [14]. The physical interpretation of $S_{p}$ is that it compares bending to frictional forces in our bead-spring chain. It bears a resemblance to a dimensionless quantity $S_{p}^{\perp}$ well-known from analytical continuum models [11,14] when substituting $6 \pi \eta a / l_{0}$ by $\gamma_{\perp}$ which is the perpendicular friction constant per unit length of a slender body. Note that $6 \pi \eta a / l_{0}$ approximates $\gamma_{\perp}$ for our bead-spring chain when hydrodynamic interactions are neglected. Via $S_{p}=L / l_{h}$, one can assign a characteristic length to the system. In the continuum model of a slender body, the analogous quantity is the elastohydrodynamic penetration length $l_{h}^{\perp}$ [11]. An oscillation with frequency $\omega$ started at one of the ends of a sufficiently long filament penetrates the filament up to the length $l_{h}^{\perp}$. Conversely, if the filament is much shorter than $l_{h}^{\perp}$, i. e., if $L \ll l_{h}^{\perp}$, the filament oscillates like a rigid rod over its whole length.

A second important quantity is the reduced magnetic field strength,

$$
B_{s}=\frac{2 \pi^{1 / 2} a^{3} \chi N}{3 \mu_{0}^{1 / 2} l_{0} A^{1 / 2}} B,
$$

which more suitably describes the effect of the external field on the superparamagnetic filament. The number $B_{s}^{2}$ compares dipolar to bending forces and it is proportional to the magnetoelastic number introduced in Ref. [8, 15].

Finally, we obtain a reduced spring constant

$$
k_{s}=\frac{N^{2} l_{0}^{3}}{A} k .
$$

While $S_{p}$ and $B_{s}$ are experimentally accessible via the frequency $\omega$ and the magnetic field strength $B, k_{s}$ is a fixed quantity of the superparamagnetic filament itself. Therefore, we do not make explicit use of the stretching mode of the filament and we always choose a sufficiently large $k_{s}$ to keep overall length fluctuations well below $10 \%$.

In what follows, we find that in the interesting regime for fluid transport the magnetic forces of the induced dipoles dominate over the bending forces of the superparamagnetic filament. It therefore makes sense to introduce a fourth dimensionless number as the ratio of frictional to magnetic forces. In literature on magnetorheological suspensions, it is also called the Mason number [45, 46]:

$$
M_{a}=S_{p}^{4} / B_{s}^{2} .
$$

\subsection{Actuation of the filament}

The direction of the actuating magnetic field of strength $B$ oscillates in the $y z$ plane (see Fig. (2):

$$
\boldsymbol{B}(t)=(0, B \sin \varphi(t), B \cos \varphi(t)),
$$




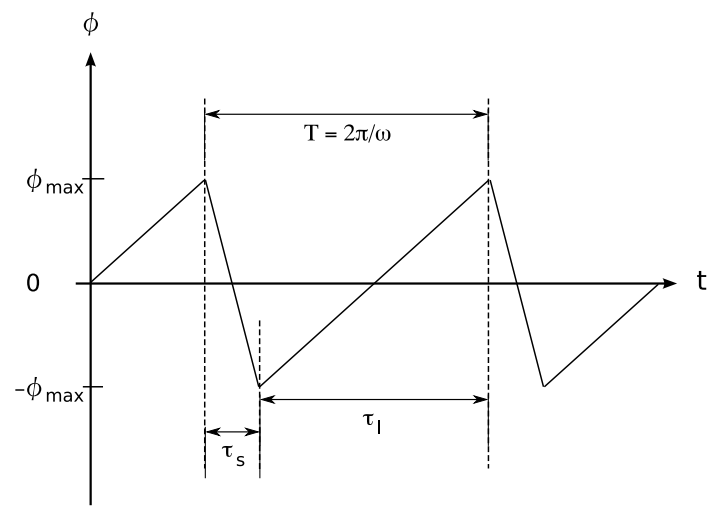

Fig. 2. The angle $\varphi$ enclosed by the magnetic field $\boldsymbol{B}(t)$ and the $z$ axis is shown as a function of time. $\varphi$ has different velocities when decreasing and increasing. The relative difference between $\tau_{s}$ and $\tau_{l}$ defines the asymmetry parameter $\varepsilon=\left(\tau_{l}-\tau_{s}\right) /\left(\tau_{s}+\tau_{l}\right)$.

where $\varphi(t)$ is the angle the field encloses with the $z$ axis. In our modeling, the angular amplitude $\varphi_{\max }$ was always $60^{\circ}$. The magnetic field induces a motion of the filament caused by the time-dependent dipole-dipole interaction of the filament's constituent beads.

In order to accomplish net fluid transport, a non-reversible motion of the filament is required [5]. In addition, the motion of the filament in positive and negative $y$ direction has to be asymmetric to achieve fluid transport along the $y$ axis. In nature, the beating cycle of biological cilia can typically be broken down into a power or transport stroke, during which the cilium moves in a fashion similar to a rigid rod with high hydrodynamic resistance, and a recovery stroke, where it bends strongly and moves back with reduced resistance close to the surface. We mimic this behavior by introducing a faster stroke in one direction followed by a slower stroke back to the original position to complement the beating cycle 47. However, in contrast to real cilia the slower stroke of our filament is the transport ('power') stroke and the faster stroke serves as the 'recovery' with a bent filament. We will illustrate this below.

To accomplish the desired asymmetry, we simply rotate the $\boldsymbol{B}$ vector fast from one side to the other and move it back more slowly. Let $T=2 \pi / \omega$ be the duration of a full actuation cycle. As illustrated in Fig. 2, we split $T$ into two parts: $T=\tau_{s}+\tau_{l}$. During the fast stroke with duration $\tau_{s}, \varphi$ is decreasing with velocity $-2 \varphi_{\max } / \tau_{s}$ and $\varphi$ is increasing during the slow stroke with duration $\tau_{l}$ with velocity $2 \varphi_{\max } / \tau_{l}$. To quantify the asymmetry in the actuation cycle and therefore in the beating pattern of the filament, we define the asymmetry parameter

$$
\varepsilon=\frac{\tau_{l}-\tau_{s}}{\tau_{s}+\tau_{l}}
$$

which is zero for $\tau_{s}=\tau_{l}$ and tends to one in the limit of $\tau_{l} \gg \tau_{s}$.

In comparison with biological cilia or the work of Kim et al. [17, the magnetic actuation technique has the advantage of not requiring any active elements within the filament or its base, such as a driving motor with a geometrical switch for force reversal. Indeed, it entirely suffices to manipulate the more easily accessible macroscopic control field, whose time protocol allows us to achieve an asymmetric and non-reciprocal beating cycle.

\section{Measuring the pumping performance}

While a non-reciprocal and asymmetric motion of the filament is required to generate a net fluid transport, a quantity to measure the pumping performance of the filament is far from obvious. In the following we will introduce such a measure based on the works in Refs. [17,48].

The main idea for the pumping performance is to integrate the fluid flow initiated by the beating filament over a whole plane parallel to the bounding surface. The integrated fluid flow is determined by the laterally averaged Blake tensor which assumes a particularly simple form 48]:

$$
\begin{aligned}
\bar{G}\left(z, z^{\prime}\right) & =\int d x d y G_{y y}^{\text {Blake }}\left(x, y, z, z^{\prime}\right) \\
& =\frac{z+z^{\prime}-\left|z-z^{\prime}\right|}{2 \eta}=\frac{\min \left(z, z^{\prime}\right)}{\eta}
\end{aligned}
$$

where we restricted ourselves to the $y y$ component since fluid is transported along the $y$ direction. Then, the integrated flow $\mathcal{F}$ generated by all the beads of the filament is approximated by [4]

$$
\mathcal{F}(z)=\frac{1}{2 \eta} \sum_{i=0}^{N}\left(z+z_{i}-\left|z-z_{i}\right|\right) F_{y i}
$$

where $z_{i}$ is the distance between the wall and bead $i$ and $F_{y i}$ is the force acting on it in $y$-direction. This expression is a function of the distance $z$ from the wall and it is constant for $z>z_{i}(i=1 \ldots N)$. For our beating filament, $\mathcal{F}(z)$ changes with time but is cyclic over a full beating cycle of the filament as is illustrated in Fig. 3 a) for $z>L$. Figure $3 \mathrm{~b}$ ) shows $\mathcal{F}(z)$ as a function of $z$ at different points of time during a typical beating cycle. As expected, $\mathcal{F}(z)$ only depends on $z$ roughly over the length $L$ of the filament and then becomes constant. Now, we consider the time average of $\mathcal{F}(z>L)$ over one beating cycle as a suitable measure for fluid transport, which we call pumping performance:

$$
\overline{\mathcal{F}}_{\infty}=\frac{1}{T} \int_{t}^{t+T} d t^{\prime} \mathcal{F}\left(z>L, t^{\prime}\right),
$$

where $T$ is the period of one actuation cycle.

To understand how well the magnetically actuated filament transports fluid, we compare it to an idealized stroke of a rigid rod with the same length parameter $L=(N-$ 1) $l_{0}$ and the same thickness as the filament (see Fig. 4):

1. In the transport stroke the rod is oriented perpendicular to the bounding surface and it is dragged parallel to the surface along a distance $L$ keeping its center a distance $L / 2+3 a$ above the surface. 


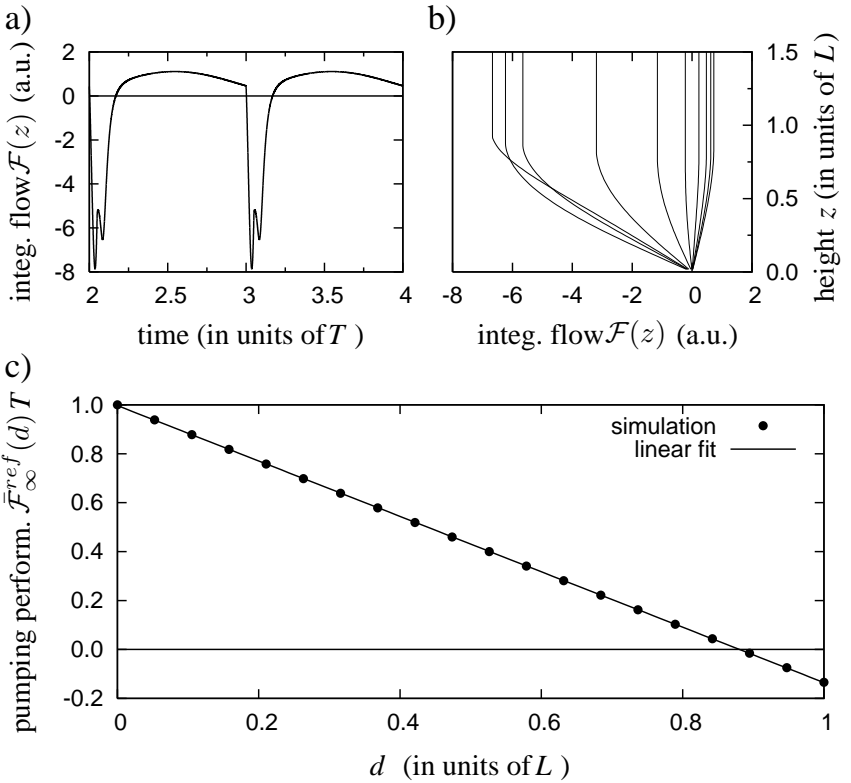

Fig. 3. a) Integrated flow $\mathcal{F}(z>L)$ in arbitrary units as a function of time in units of $T$ for the parameters $S_{p}=3, B_{s}=3$ and $\varepsilon=0.9$. b) Integrated flow $\mathcal{F}(z=1.5 L)$ as a function of height $z$ at different points of time during one beating cycle. The stroboscopic snapshots to the right correspond to the slow part of the stroke and the ones to the left to the faster stroke. c) Pumping performance $\overline{\mathcal{F}}_{\infty}^{\text {ref }}(0)$ times period $T$ of the idealised reference cycle as a function of the parameter $d$ as defined by Eq. (29). The $y$ axis is scaled to units of the maximal value $\overline{\mathcal{F}}_{\infty}^{\text {ref }}(0)=3.895 \times 10^{-14} \mathrm{~m}^{3} / \mathrm{s}$ at $d=0$.
1) transport stroke

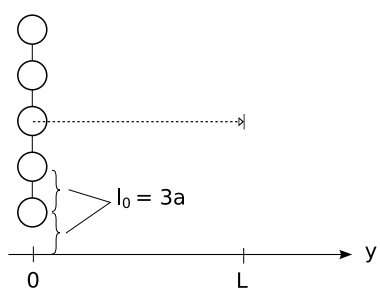

2) recovery stroke

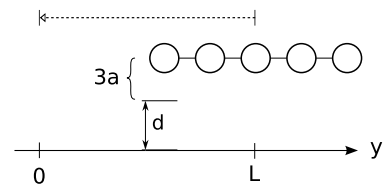

Fig. 4. Geometry of the idealized stroke of a rigid rod consisting of $N$ particles of radius $a$ with distance $l_{0}$ between the centers of adjacent beads. The length parameter $L$ is defined as $L=(N-1) l_{0}$

2. The rod is then rotated by $90^{\circ}$ to be parallel to the surface and then in the recovery stroke it is dragged along its long axis to its original position with a separation of $d+3 a$ above the surface.

In unbounded Stokes flow, the first and second part of the stroke use the respective maximal and minimal hydrodynamic resistance to produce a net fluid transport over one cycle. In the presence of a no-slip wall, the hydrodynamic resistance increases closer to the wall. Therefore, the distance $d$ in the second part turns out to be an important parameter.
For the idealized stroke, we define a reference pumping performance

$$
\overline{\mathcal{F}}_{\infty}^{r e f}(d)=\frac{1}{T} \int_{t}^{t+T} d t^{\prime} \mathcal{F}\left(z>L, t^{\prime}\right)
$$

where $T=T_{\perp}+T_{\|}$is the time required for both parts of the cycle and the variable $d$ emphasizes the separation between the rigid rod and the surface for the second part of the reference cycle. We determined the reference pumping performance with the help of our filament as follows. We let a constant force act on each bead and numerically evaluated the average velocity of the beads in both parts of the strokes from which we then calculated the times $T_{\perp}$ and $T_{\|}$the filament takes to move the length $L$. Together with Eq. (27) $\overline{\mathcal{F}}_{\infty}^{r e f}(d)$ is then determined. In particular, it becomes clear that $\overline{\mathcal{F}}_{\infty}^{\text {ref }}(d) T$ only depends on effective friction coeffcients, $L$, and $d$. Note that in both parts of the reference stroke, the filament experiences an additional torque which alters its orientation. To really realize the idealized stroke, one would have to counterbalance the frictional torques appropriately. Fig [3.) shows $\overline{\mathcal{F}}_{\infty}^{\text {ref }}(d) T$ as a function of $d$. A linear fit nicely connects the data points acquired by simulations and represents the linear dependence of $\mathcal{F}\left(z>L, t^{\prime}\right)$ on $d$ in the recovery stroke. It makes sense that maximal pumping occurs when the recovery stroke of the reference cycle is at a minimum distance $3 a$ from the wall $(d=0)$ because the no-slip boundary condition reduces the fluid volume the rod can drag along. Interestingly, a reversal of the pumping direction sets in at about $d=0.9$. Here, the perpendicular movement of the rod gives rise to less fluid transport compared to the parallel one simply because it is on average much closer to the boundary. This highlights the importance of hydrodynamic interactions not only amongst the beads but also with the bounding wall.

The reference stroke describes an optimum fluid transport one can achieve with a rigid rod of length $L$ close to a surface. We therefore compare the pumping performance $\overline{\mathcal{F}}_{\infty}$ of the magnetically actuated filament to the reference value $\overline{\mathcal{F}}_{\infty}^{\text {ref }}(d=0)$ at $d=0$ i.e., when the idealized reference cycle assumes ist maximum value. This results in the reduced pumping performance

$$
\xi=\frac{\overline{\mathcal{F}}_{\infty}}{\overline{\mathcal{F}}_{\infty}^{\text {ref }}(d=0)},
$$

which we use in the following. Expression $\overline{\mathcal{F}}_{\infty}$ in Eq. 30 is evaluated after the filament has assumed its limiting cycle, which typically happens after less than five actuation cycles after starting the simulation. We have checked that $\xi$ evaluates to zero either if the motion of the filament is symmetric $\left(\tau_{s}=\tau_{l}\right)$ or if it is reciprocal, i.e., if the filament does not bend, e.g., in the case $S_{p} \rightarrow 0$. Hence, Eq. (30) constitutes a suitable measure for characterizing the pumping performance of the magnetically actuated artificial cilium. 
a)

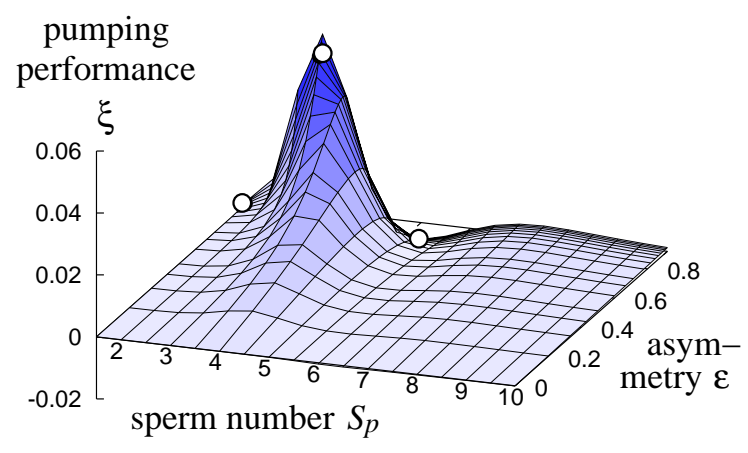

b)

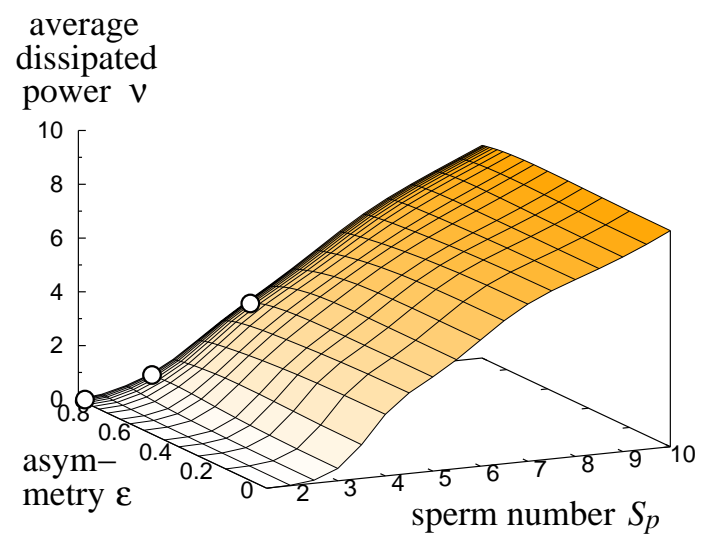

Fig. 5. Reduced pumping performance $\xi(\mathrm{a})$ and the timeaveraged dissipated power $\nu$ in arbitrary units (b) for a single cilium as a function of sperm number $S_{p}$ and asymmetry parameter $\varepsilon$. The reduced magnetic field strength is $B_{s}=2.5$. The white dots mark parameters for which stroboscopic snapshots of the filament are shown in Fig. 6a).

\section{Pumping performance of a single artificial cilium}

Figure 5a) shows the pumping performance $\xi$ of a single filament as a function of the Sperm number $S_{p}$ and the asymmetry parameter $\varepsilon$ at a fixed strength of the magnetic field $B_{s}=2.5$. The most striking feature is that the performance is strongly peaked for $\varepsilon$ close to one and at $S_{p} \approx 3$. Such a peak is also observed in the swimming velocity of the artificial micro-swimmer 8, 15, 18. The corresponding stroke pattern for $S_{p} \approx 3$ is illustrated in the middle picture of Fig. 6a). In the slow transport stroke the filament rotates clockwise being nearly straight. It uses the high friction coefficient of a rigid rod dragged perpendicular to its axis to pump fluid. In the fast recovery stroke, the filament bends due to the large hydrodynamic friction forces that scale with velocity and then relaxes back to the initial configuration. As Fig. 3a) illustrates, fluid transport is also noticeable in the recovery stroke. So, the pumping performance, even for the most efficient stroke pattern, is the result of a small asymmetry in the amount of fluid transported to the right or left. In the example of Fig. [3a), which is close to the optimum stroke pattern, only $4.3 \%$ of the total amount of moved fluid are effectively transported in positive $y$ direction. As a result, the maximum pumping performance in Fig. 5 a) is only $6 \%$ of the reference stroke. As expected, the pumping performance vanishes for symmetric beating about the $z$ axis, i.e., when $\varepsilon=0$. The same is true for $S_{p} \rightarrow 0$ : The filament follows the actuating magnetic field instantaneously. Aside from the base, the filament therefore remains straight and the stroke is reciprocal. The left picture in Fig. 6a) gives an example of such a stroke. A reversal of the pumping direction $(\xi<0)$ occurs at $S_{p} \approx 5.5$, albeit only with a rather weak performance. Finally, the pumping performance goes to zero for increasing $S_{p}$ or frequency since the filament can no longer follow the actuating field as illustrated in the right picture of Fig. 6a). In summary, an optimal pumping performances is only achieved for intermediate values of $S_{p}$.

Let us add a further remark. Naively, one might anticipate that the closer $\varepsilon$ is to one, the more pronounced the incurred bending during the fast part of the actuation cycle, giving rise to better pumping performance. However, a limit on the maximal speed with which the filament manages to follow the field exists. The extreme case of instantaneous switching of the direction of the magnetic field in the recovery stroke $\left(\tau_{s}=0\right)$ is not conducive to generating fluid transport. On the contrary, it seems important that the free end of the filament follows the magnetic field's orientation during the fast stroke. Relaxation from the bent to a straight configuration then happens during the time interval $\tau_{l}$ allotted to the slow part of the cycle. Consequently, the filament takes significantly longer to perform the actual recovery stroke of the cycle than the magnetic field. Naturally, this observation translates into the fact that the $\varepsilon$ value at which maximum pumping performance occurs decreases with increasing $S_{p}$. In Fig. 5 a), where $\varepsilon$ ranges up to 0.94 this is visible to the right of the optimal pumping performance.

Part b) of Fig. [5] shows the time average of the dissipated power,

$$
\nu=\frac{1}{T} \int_{t}^{t+T} \sum_{i=0}^{N} \boldsymbol{v}_{i} \cdot \boldsymbol{F}_{i} d t^{\prime}
$$

as a function of $S_{p}$ and $\varepsilon$. Surprisingly, it does not display such a pronounced behavior as the pumping performance. Particularly, there is hardly any dependence on the asymmetry parameter $\varepsilon$ visible. Being proportional to the square of the beads' velocities, one could assume $\nu \sim \omega^{2}$ and therefore $\nu \sim S_{p}^{8}$ (recall that $S_{p} \sim \omega^{1 / 4}$ ). At small $S_{p}$, the data shown in Fig. 50) do show a steep incline. However, they do not increase as $S_{p}^{8}$, because already at $S_{p}$ around 2 the filament does not completely follow the actuating field as the snaphots in Fig. 6a) illustrate. This effect becomes more pronounced for increasing $S_{p}$, and the data clearly deviate from the naive assumption of $\nu \sim S_{p}^{8}$. The third picture in Fig. 6a) demonstrates that already at $S_{p}=5$ the filament lags significantly behind the actuating magnetic field. 
a) fixed $B_{S}=2.5$

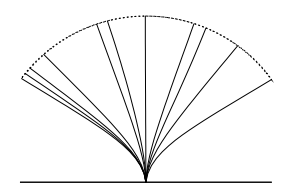

$S_{p}=1.5$

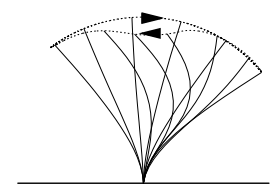

$S_{p}=3$

b) fixed $S_{p}=3$
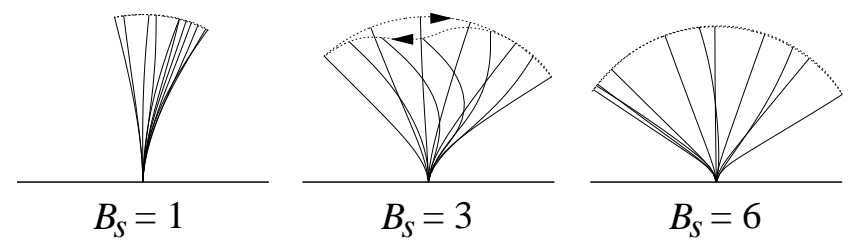

Fig. 6. Stroboscopic snapshots of the filament at different times during the beating cycle for $\varepsilon=0.9$. The trajectory of the top bead during one beating cycle is also indicated. In the slow transport stroke the filament rotates clockwise, the fast recovery stroke occurs to the left, as indicated by the arrows. A pronounced bending of the filament occurs only at intermediate sperm number $S_{p}$ and magnetic field strength $B_{s}$.

As illustrated by the three-dimensional plot of Fig. [7 ), there exists a pronounced dependence of the pumping performance $\xi$ on the strength $B_{s}$ of the actuating magnetic field which has also been observed in the swimming velocity of the artificial micro-swimmer [8,15, 18. Figure 7b) shows this behavior for different constant values of $S_{p}$. Increasing the magnetic field from zero, the pumping perfomance stays close to zero and then, beyond a certain threshold value, it grows until it reaches a maximum. It finally decreases and even becomes negative. The snapshots in Fig. 6b) again explain this behavior. Small field strengths $B_{s}$ are not high enough to overcome the hydrodynamic friction forces and therefore the motion of the filament is very limited. On the other hand, at large strengths $B_{s}$ the filament is always straight and therefore performs a reciprocal motion. An optimal stroke only exists in an intermediate regime for the strength $B_{s}$. Clearly, the optimal performance shifts with increasing $S_{p}$ to larger values of $B_{s}$ since a larger field is needed to move the filament through the fluid. In other words, for larger $B_{s}$ the filament is stiffer and a higher frequency $\omega \propto S_{p}^{4}$ is needed to achieve the optimum stroke. Higher frequency means larger frictional forces and therefore a larger pumping performance, as Figs. (7a) and b) demonstrate. When the magnetic forces on the filament exceed the bending forces, one expects the dynamics of the filament to be determined by the ratio of the hydrodynamic friction to magnetic forces, which we introduced in Eq. (23) as Mason number $M_{a}$. From Fig. 7 7 ) we extract curves $\xi\left(S_{p}\right)$ for different constant $B_{s}$, and rescale each of these curves with the respective maximum values $\xi_{m}$. In Fig. (7)), all the data points for $B_{s} \geq 2$ are plotted as a function of the Mason number $M_{a}$. Indeed, most of the data points fall on a master curve, as predicted. Deviations occur for data points with $B_{s}$ close to 2 . a)

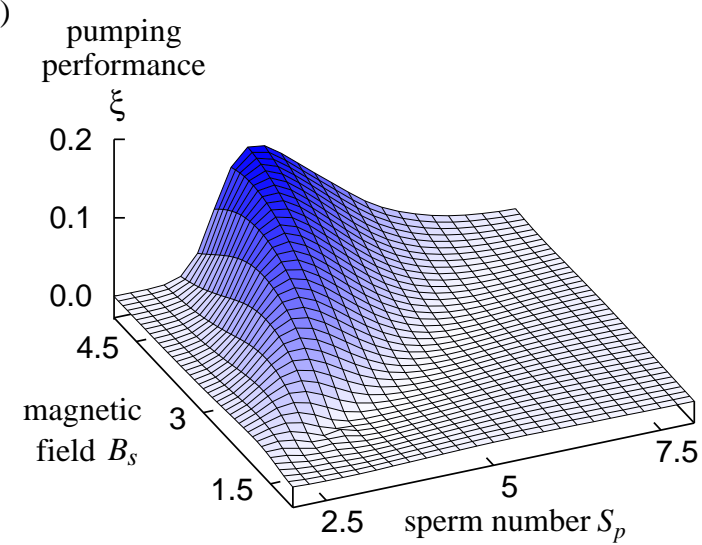

b)

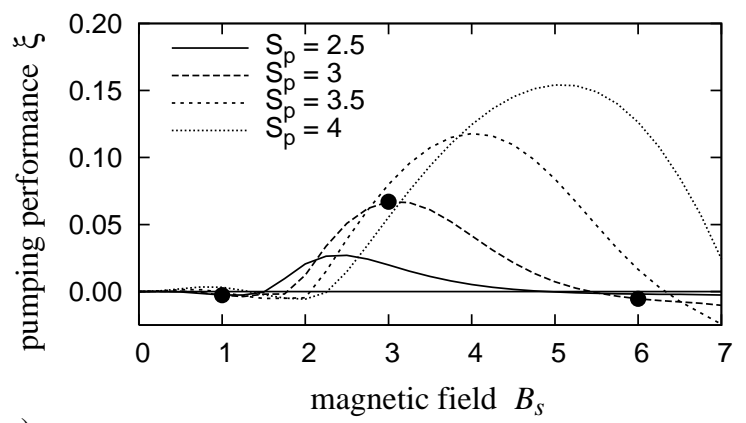

c)

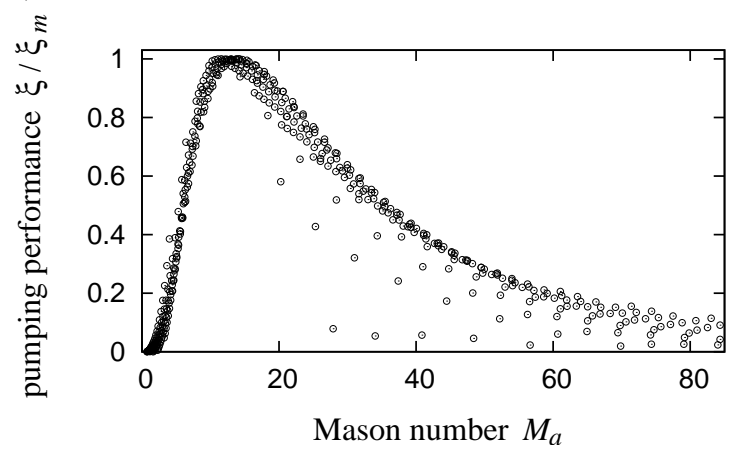

Fig. 7. a) Pumping performance $\xi$ as a function of $S_{p}$ and $B_{s}$ for $\varepsilon=0.9$. b) Plot of $\xi$ versus $B_{s}$ for different $S_{p}$. The black dots mark parameters for which stroboscopic snapshots of the filament are shown in Fig. 6b). c) Pumping performance $\xi$ as a function of the Mason number $M_{a}=S_{p}^{4} / B_{s}^{2}$, where $\xi$ is given in units of the maximum values $\xi_{m}$ when $B_{s}$ is kept constant.

\section{Pumping performance of hydrodynamically interacting cilia}

Nature often uses arrays of beating cilia rather then a single isolated cilium for generating fluid transport or to propel microorganisms such as a paramecium [2]. These cilia beat in a synchronized fashion with a defined phase difference giving rise to so-called metachronal waves. As reviewed in the introduction, analytical and numerical investigations of beating cilia suggest that hydrodynamic interactions between single cilia synchronize their beating by inducing such a phase lag and therefore waves to occur. For example, in Ref. [17. model cilia are driven 


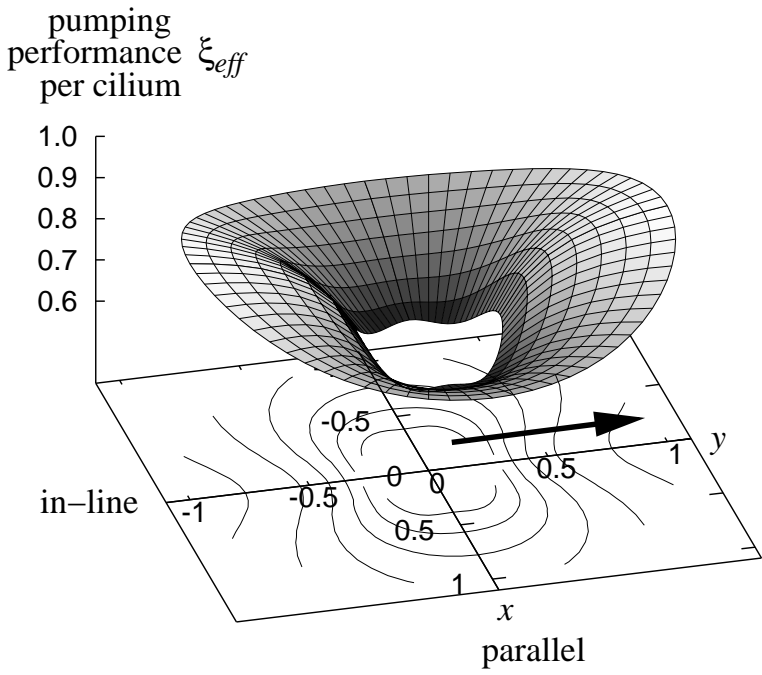

Fig. 8. Effective pumping performance $\xi_{\text {eff }}$ for a two-cilia system. One cilium is placed at $x=y=0$, the other cilium at different points in the $x y$ plane with coordinates in units of $L$. The effective pumping performance per cilium, $\xi_{\text {eff }}$, is given in units of the performance $\xi$ for a single, unperturbed filament actuated with the same parameters $S_{p}=3, B_{s}=3$ and $\varepsilon=0.9$. The base contour lines run from 0.4 (innermost) to 0.9 (outermost) in steps of 0.1 . The arrow indicates the pumping direction along the $y$ axis.

by imposing a torque at the base of each cilium. This torque is reversed once the angular amplitude of the cilium close to the base exceeds a certain threshold value. Due to this mechanism, the phase of the driving torque and therefore of each cilium can change during the beating cycle and a phase lag between neighboring cilia can evolve. This cannot happen for the magnetically actuated cilia studied in this paper since the oscillating magnetic field determines the phase of each cilium. Nevertheless, one might ask if in our system the zero-phase-lag state is stable. We have never observed an instability towards a state with non-zero phase lag. This seems to be reasonable: the actuating magnetic field ultimately determines the forces which drive the filament against the hydrodynamic friction through the liquid. Since these forces are much larger than the hydrodynamic interactions between the cilia, a noticable phase lag between the cilia cannot occur. On the other hand, the artificial cilia system can be used to investigate in a systematic way how the pumping performance depends on the phase lag between the cilia by actuating each cilium with a separate magnetic field. In the following, we present initial results of such a study.

In Fig. 8 we first investigate the pumping performance of a two-cilia system with zero phase lag between the cilia. One cilium is placed at the center $x=y=0$ and the other at different points in the $x y$ plane. We introduce an effective pumping performance per cilium, $\xi_{\text {eff }}$, in units of the performance $\xi$ of a single cilium actuated with the same magnetic field cycle. If the cilia are sufficiently well separated from each other, $\xi_{\text {eff }}$ approaches the pump- a)

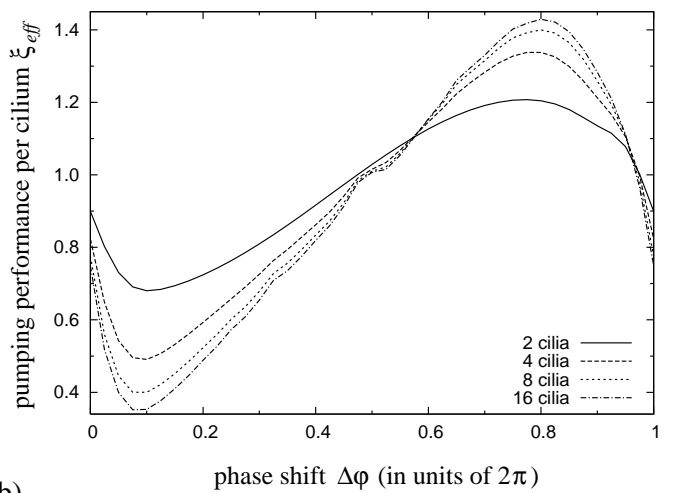

b)

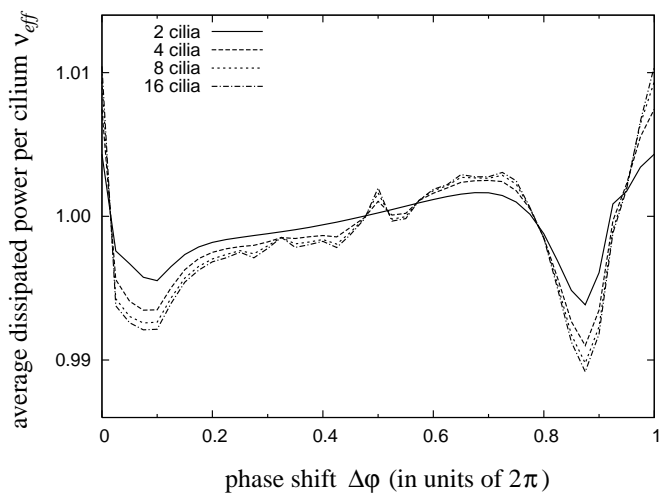

Fig. 9. a) Average effective pumping performance $\xi_{\text {eff }}$ for multi-cilia systems in the in-line configuration as a function of the phase shift between neighboring cilia. $\xi_{\text {eff }}$ is given in units of the performance $\xi$ for a single, unperturbed filament actuated with the same parameters $S_{p}=3, B_{s}=3$ and $\varepsilon=0.9$. b) Average dissipated power $\nu_{\text {eff }}$ for multi-cilia systems in the in-line configuration as a function of the phase shift between neighboring cilia. $\nu_{\text {eff }}$ is given in units of the dissipated power $\nu$ for a single, unperturbed filament.

ing performance of a single filament, as expected. If the cilia approach each other, $\xi_{\text {eff }}$ decreases. Due to hydrodynamic interactions between the cilia beating with the same phase, their effective friction with the surrounding fluid is reduced and therefore they pump less fluid. Figure 8 clearly shows that this effect is more pronounced in the parallel configuration, where the cilia are placed next to each other on the $x$ axis while the strokes are performed in the $y z$ plane. On the other hand, in the in-line configuration, where the cilia sit behind each other on the $y$ axis, $\xi_{\text {eff }}$ has almost reached the single-cilium performance $\xi$ at a distance $L$.

Next we study the pumping performance and the dissipated energy of several cilia placed on the $y$ axis in the in-line configuration. Neighboring cilia are actuated by different magnetic fields with a prescribed phase shift $\Delta \varphi$. As illustrated by the first row in Fig. 10a), a cilium lags behind its neighboring cilium to the left by the small phase shift $\Delta \varphi$. Note that the slow transport stroke with a straight cilium goes to the right as can be seen from the snapshots at different times in Fig. [10a). Furthermore, when time proceeds, the strongly bent cilium in the 
recovery stroke also moves to the right. So metachronal wave propagation and transport stroke occur in the same direction which is also termed symplectic metachronism in literature 2. However, note that in biological systems the transport stroke is faster than the recovery stroke. On the other hand, for phase shifts close to $2 \pi$ [see Fig. [10b)] a cilium moves ahead of its neighbor to the left. As a result, the metachronal wave propagates opposite to the transport stroke and is therefore termed antiplectic. Figure 9a) demonstrates that the average effective pumping performance per cilium, $\xi_{\text {eff }}$, falls below the reference value of a single cilium for small $\Delta \varphi$ while it assumes a maximum value at around $\Delta \varphi=0.8 \times 2 \pi$, i.e., in the antiplectic mode. The maximal pumping performance $\xi_{\text {eff }}$ increases with the number of cilia and slightly moves to larger $\Delta \varphi$. Even at the relatively large distance of $1.5 \mathrm{~L}$ between the cilia, $\xi_{\text {eff }}$ is increased by more than $40 \%$ relative to a single cilium. Preliminary calculations show that this value strongly increases, when the cilia are moved closer together. Hence, our study clearly demonstrates that metachronal coordination of ciliary beating at the right phase shift significantly enhances the ability to transport fluid. An explanation for this behavior can be inferred from the snapshots in Fig. 10, In the optimum stroke [see Fig. [10b)], the fifth cilium from left performs the recovery stroke against the neighboring fourth cilium which hinders the fluid flow initiated by recovery stroke and therefore increases fluid transport to the right. On the other hand, in the metachronal wave with lowest pumping performance [see Fig. [10 $)$ ], the cilium performing the recovery stroke is further away from the neighboring cilia and therefore its fluid transport is hardly hindered. While the pumping performance is strongly influenced by the phase shift and the number of beating cilia, the average dissipated power per cilium, $\nu_{e f f}$, only exhibits very weak dependence on these parameters. As illustrated in Fig. 9b), $\nu_{\text {eff }}$ deviates from the single-cilium value by at most $1 \%$. This is in contrast to the work of Gueron and LevitGurevich, who, e.g., found that for a 10-cilia system with a distance of $0.7 \mathrm{~L}$ between the cilia $\nu_{\text {eff }}$ decreases by ca. 40 $\%$ relative to the value of a single cilium [21]. We checked that the smaller distance is not the major reason for this difference. It might be due to the fact that these authors model biological cilia with their internal actuation mechanism, whereas our cilia are actuated by an external field.

\section{Conclusion}

We have performed a thorough theoretical investigation of a magnetically powered low Reynolds number pumping device. It consists of a superparamagnetic elastic filament that is attached to a surface and that can be conveniently actuated by an external magnetic field. We have introduced a reduced pumping performance $\xi$ that quantifies the fluid transport and compares it to the value of an idealized transport stroke of a rigid rod. With the help of $\xi$, we have identified an optimum stroke pattern of the magnetically actuated artificial cilium. It consists of a slow transport stroke and a fast recovery stroke in contrast a) $\Delta \varphi=0.08 * 2 \pi$

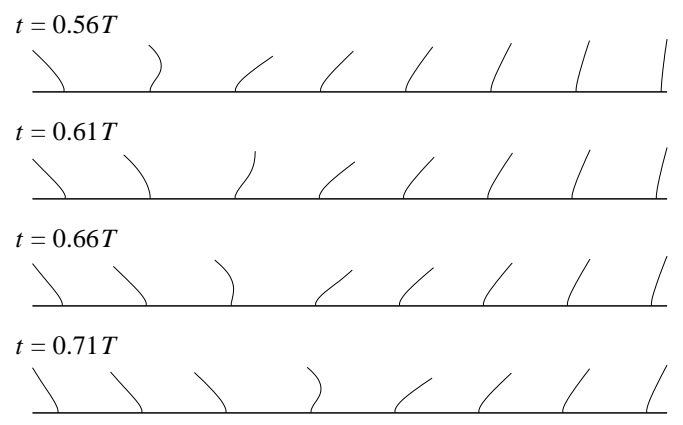

b) $\Delta \varphi=0.8 * 2 \pi$

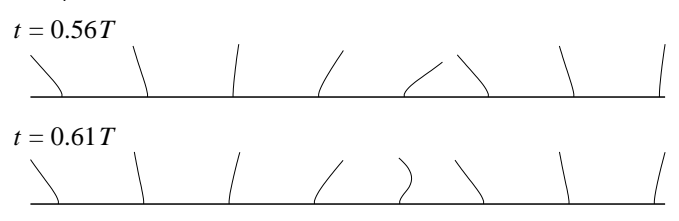

$t=0.66 T$

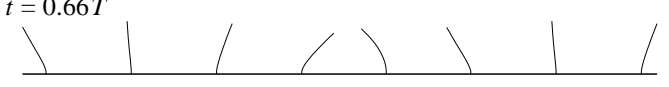

$t=0.71 T$

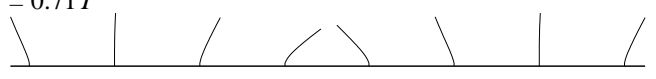

Fig. 10. Snapshots of the in-line configuration with eight cilia for different phase shifts; a) $\Delta \varphi=0.08 \times 2 \pi$ (minimal pumping performance) and b) $\Delta \varphi=0.8 \times 2 \pi$ (optimum pumping performance). The times given in units of the time period $T$ belong to the field cycle of the rightmost cilium.

to nature. Our detailed parameter study for the reduced magnetic field $B_{s}$ and the sperm number $S_{p}$ reveals that for sufficiently large $B_{s}$ the behavior of the artificial cilium is mainly governed by the Mason number that compares frictional to magnetic forces. Initial studies on multi-cilia systems reveal that the pumping performance is very sensitive to the imposed phase lag between neighboring cilia, i.e., to the details of the initiated metachronal waves. Due to our results, we expect that metachronal waves in real cilia systems also increase the pumping performance for fluid transport.

Experiments using the superparamagnetic elastic filament for fluid transport are currently under way [50]. Our study will help to identify relevant parameter ranges in which the artifical cilium can be operated optimally. Initiating metachronal waves in the multi-cilia system will be important for increasing the pumping performance. This requires oscillating magnetic fields the phases of which should vary from one cilium to the other. The realization of such a system is not unrealistic [51] but certainly poses a challenge to experimentalists. It would help to establish the artificial cilium as a useful tool for fluid transport in microfluidic devices.

The authors would like to thank J. Bibette, R. Dreyfus, M. Fermigier, G. Maret, M. Reichert, and H. Stone for engaging and interesting discussions. EMG and HS thank the International Graduate College at the University of Konstanz for financial 
support and the MPI for Dynamics and Self-Organization in Göttingen for providing us with computing time. EMG acknowledges support from the Marie Curie Early Stage Training network QIPEST (MEST-CT-2005-020505). MD and HS acknowledge financial support from the Deutsche Forschungsgemeinschaft under Grant No. Sta 352/7-1.

\section{A Simulation Parameters}

In table 1, we summarize the parameters used for the numerical simulations. The oscillation frequency $\omega=2 \pi / T$ and the magnetic-field strength $B$ were varied to study the respective ranges of sperm number $S_{p}$ and reduced magnetic field $B_{s}$. The spring constant $k$, the time step $\Delta t$ for the Euler integration, and the number $n_{s}$ of simulation cycles were adjusted as necessary. Typical values are shown.

\section{B Mobilities}

Explicit expressions for the stresslet and sourcelet contricutions of Blake's tensor can be calculated from Ref. 33. or taken directly from Ref. 52. Starting with these, we have calculated the self- and cross-mobilities as outlined in the main text.

\section{B.1 Self mobilities}

Due to the axial symmetry of the sphere with its image system all but the diagonal elements vanish and the $x x$ and the $y y$ elements are identical:

$$
\delta \boldsymbol{\mu}_{\text {self }}=\frac{1}{6 \pi \eta a}\left(\begin{array}{ccc}
\nu & 0 & 0 \\
0 & \nu & 0 \\
0 & 0 & 2 \nu
\end{array}\right)
$$

where $\nu$ is given by (with $a$ being the radius of the bead and $z$ the distance to the plane)

$$
\nu=-\frac{3}{16}\left[\frac{a}{z}-\left(\frac{a}{z}\right)^{3}+\frac{1}{3}\left(\frac{a}{z}\right)^{5}\right] .
$$

Once the expression above is included into formula (18) for $\boldsymbol{\mu}_{i i}$, the self-mobility agrees with the expression given in Ref. [41.

\section{B.2 Cross mobilities}

Symmetry dictates that the $x y$ and $y x$ elements are identical (the boundary wall is in the $x y$ plane). Furthermore, the $x x$ and $y y$ elements are formally equivalent when $R_{x}$ is interchanged with $R_{y}$. The same holds for the $x z$ and $y z$ or $z x$ and $z y$ elements, respectively.

The positions of the beads are $\boldsymbol{r}_{1}=\left(x_{1}, y_{1}, z_{1}\right)$ and $\boldsymbol{r}_{2}=\left(x_{2}, y_{2}, z_{2}\right)$ and the image system is located at $\overline{\boldsymbol{r}}_{2}=$ $\left(x_{2}, y_{2},-z_{2}\right)$. Furthermore, for convenience we define $s=$ $\left|\boldsymbol{r}_{1}-\overline{\boldsymbol{r}}_{2}\right|$ and $R_{x}=\left(x_{1}-x_{2}\right), R_{y}=\left(y_{1}-y_{2}\right)$ and $R_{z}=$ $\left(z_{1}+z_{2}\right)$. Let $\alpha$ and $\beta$ refer to the $x$ and $y$ but not the $z$ coordinate. In this notation, we obtain the following components for the cross mobilities:
Table 1. Parameters used in the numerical studies.

\begin{tabular}{ll}
\hline Parameter & Simulation value \\
\hline \hline$N$ & 20 \\
$a[\mu \mathrm{m}]$ & 0.5 \\
$l_{0}$ & $3 a$ \\
$\rightarrow L[\mu \mathrm{m}]$ & 28.5 \\
$\chi$ & 0.993 \\
$\eta\left[\mathrm{Ns} / \mathrm{m}^{2}\right]$ & $10^{-3}$ \\
$k[\mathrm{~N} / \mathrm{m}]$ & $1.5 \cdot 10^{-3}$ \\
$A[\mathrm{Nm}]$ & $4.5 \cdot 10^{-22}$ \\
$\omega[2 \pi / s]$ & $0.071 \ldots 140.72$ \\
$\rightarrow S_{p}$ & $1.5 \ldots 10$ \\
$B[\mathrm{~T}]$ & $0 \ldots 0.085$ \\
$\rightarrow B_{s}$ & $0 \ldots 7$ \\
$\varphi_{\max }\left[{ }^{\circ}\right]$ & 60 \\
$\Delta t[\mathrm{~s}]$ & $\mathcal{O}\left(10^{-6}\right)$ \\
$n_{s}$ & $\approx 5$ \\
\hline
\end{tabular}

\section{References}

1. J. den Toonder, F. Bos, D. Broer, L. Filippini, M. Gillies, J. de Groede, T. Mol, M. Reijme, W. Talen, H. Wilderbeek, V. Khatavkar, and P. Anderson, Lab Chip 8, 533 (2008).

2. C. Brennen and H. Winet, Ann. Rev. Fluid Mech. 9, 339 (1977).

3. R. W. Linck, Cilia and Flagella in Encyclopedia of Life Sciences (Wiley, Chichester, 2001), www.els.net.

4. D. Bray, Cell Movements: From Molecules to Motiliy, 2nd ed., Garland Publishing, New York (2001).

5. E. M. Purcell, Am. J. Phys. 45, 3 (1977).

6. I. Ibañez-Tallon, A. Pagenstecher, M. Fliegauf, H. Olbrich, A. Kispert, U.-P. Ketelsen, A. North, N. Heintz, and H. Omran, Hum. Mol. Genet. 13, 2133 (2004).

7. C. D. Stern, Nature 418, 29 (2002); J. J. Essner, K. J. Vogan, M. K. Wagner, C. J. Tabin, H. J. Jost Yost, and M. Brueckner, Nature 418, 37 (2002); S. Nonaka, H. Shiratori, Y. Saijoh, and H. Hamada, Nature 418, 96 (2002).

8. R. Dreyfus, J. Baudry, M. L. Roper, M. Fermigier, H. A. Stone, and J. Bibette, Nature 437, 862 (2005).

9. B. A. Evans, A. R. Shields, R. Lloyd Carroll, S. Washburn, M. R. Falvo, and R. Superfine, Nano Letters, 7, 1428 (2007).

10. N. Darnton, L. Turner, K. Breuer, and H. C. Berg, Biophys. J. 86, 1863 (2004).

11. C. H. Wiggins and R. E. Goldstein, Phys. Rev. Lett. 80, 3879 (1998); C. H. Wiggins, D. Riveline, A. Ott, and R. E. Goldstein, Biophys. J. 74, 1043 (1998).

12. S. Gueron and K. Levit-Gurevich, Biophys. J. 74, 1658 (1998).

13. S. Camalet, F. Jülicher, and J. Prost, Phys. Rev. Lett. 82, 1592 (1999); S. Camalet and F. Jülicher, New J. Phys. 2, 24 (2000).

14. C. P. Lowe, Phil. Trans. R. Soc. Lond. B 358, 1543 (2003); M. C. Lagomarsino, F. Capuani, C. P. Lowe, J. Theor. Biol. 222, 215 (2003).

15. M. L. Roper, R. Dreyfus, J. Baudry, M. Fermigier, J. Bibette, and H. A. Stone, J. Fluid Mech. 554, 167 (2006); Proc. Royal Soc. A 464, 877 (2008).

16. M. Manghi, X. Schlagberger, and R. Netz, Phys. Rev. Lett. 96, 068101 (2006). 


$$
\begin{aligned}
\left(\delta \boldsymbol{\mu}_{12}\right)_{\alpha \alpha} & =\frac{1}{4 \pi \eta}\left[-z_{1} z_{2}\left(\frac{1}{s^{3}}-3 \frac{R_{\alpha}^{2}}{s^{5}}\right)-\frac{a^{2}}{s^{7}} R_{z}^{2}\left(4 R_{\alpha}^{2}-R_{\beta}^{2}-R_{z}^{2}\right)\right. \\
& \left.-\frac{a^{4}}{3 s^{9}}\left(4 R_{\alpha}^{4}-R_{\beta}^{4}+4 R_{z}^{4}+3 R_{\alpha}^{2} R_{\beta}^{2}+3 R_{\beta}^{2} R_{z}^{2}-27 R_{\alpha}^{2} R_{z}^{2}\right)\right] \\
\left(\delta \boldsymbol{\mu}_{12}\right)_{z z} & =\frac{1}{4 \pi \eta}\left[z_{1} z_{2}\left(\frac{1}{s^{3}}-3 \frac{R_{z}^{2}}{s^{5}}\right)-\frac{a^{2}}{s^{7}} R_{z}^{2}\left(3\left(R_{\alpha}^{2}+R_{\beta}^{2}\right)-2 R_{z}^{2}\right)\right. \\
& \left.-\frac{a^{4}}{3 s^{9}}\left(3 R_{\alpha}^{4}+3 R_{\beta}^{4}+6 R_{\alpha}^{2} R_{\beta}^{2}-24 R_{z}^{2}\left(R_{\alpha}^{2}+R_{\beta}^{2}\right)+8 R_{z}^{4}\right)\right] \\
\left(\delta \boldsymbol{\mu}_{12}\right)_{\alpha \beta} & =\frac{1}{4 \pi \eta}\left[\frac{3 z_{1} z_{2} R_{\alpha} R_{\beta}}{s^{5}}-\frac{5 a^{2}}{s^{7}} R_{\alpha} R_{\beta} R_{z}^{2}-\frac{5 a^{4}}{3 s^{9}}\left(R_{\alpha}^{2}+R_{\beta}^{2}-6 R_{z}^{2}\right) R_{\alpha} R_{\beta}\right] \\
\left(\delta \boldsymbol{\mu}_{12}\right)_{\alpha z} & =\frac{1}{4 \pi \eta}\left[R_{\alpha}\left(\frac{z_{1}^{2}}{s^{3}}-3 \frac{z_{1} z_{2} R_{z}}{s^{5}}\right)+R_{\alpha} a^{2}\left(\frac{1}{3} \frac{1}{s^{3}}+\frac{5}{s^{7}} R_{z}^{3}-\frac{1}{s^{5}}\left[R_{z}+2\left(z_{1}^{2}+z_{1} z_{2}\right)\right]\right)\right. \\
& \left.+\frac{5 a^{4}}{3 s^{9}} R_{\alpha}\left(3 R_{z}\left[R_{\alpha}^{2}+R_{\beta}^{2}\right]-4 R_{z}^{3}\right)\right] \\
\left(\delta \boldsymbol{\mu}_{12}\right)_{z \alpha} & =\frac{1}{4 \pi \eta}\left[R_{\alpha}\left(\frac{z_{1}^{2}}{s^{3}}+3 \frac{z_{1} z_{2} R_{z}}{s^{5}}\right)+R_{\alpha} a^{2}\left(\frac{1}{3} \frac{1}{s^{3}}-\frac{5}{s^{7}} R_{z}^{3}+\frac{1}{s^{5}}\left[R_{z}-2\left(z_{1}^{2}+z_{1} z_{2}\right)\right]\right)\right. \\
& \left.-\frac{5 a^{4}}{3 s^{9}} R_{\alpha}\left(3 R_{z}\left[R_{\alpha}^{2}+R_{\beta}^{2}\right]-4 R_{z}^{3}\right)\right]
\end{aligned}
$$

17. Y. Kim, and R. Netz, Phys. Rev. Lett. 96, 158101 (2006).

18. E. Gauger, and H. Stark, Phys. Rev. E 74, 021907 (2006).

19. E. Lauga, Phys. Rev. E 75, 041916 (2007).

20. S. Gueron, K. Levit-Gurevich, N. Liron, and J. J. Blum, Proc. Natl. Acad. Sci. USA 94, 6001 (1997).

21. S. Gueron and K. Levit-Gurevich, Proc. Natl. Acad. Sci. USA 96, 12240 (1999).

22. M. C. Lagomarsino, B. Bassetti, and P. Jona, Eur. Phys. J. E 26, 81 (2002); M. C. Lagomarsino, P. Jona, and B. Bassetti, Phys. Rev. E 68, 021908 (2003).

23. A. Vilfan, and F. Jülicher, Phys. Rev. Lett. 96, 058102 (2006).

24. P. Lenz and A. Ryskin, Phys. Biol. textbf3, 285 (2006).

25. B. Guirao and J.-F. Joanny, Biophys. J. 92, 1900 (2007).

26. J. Elgeti and G. Gompper, in preparation

27. C. Goubault, P. Jop, M. Fermigier, J. Baudry, E. Bertrand, and J. Bibette, Phys. Rev. Lett. 91, 260802 (2003).

28. L. Cohen-Tannoudji, E. Bertrand, L. Bressy, C. Goubault, J. Baudry, J. Klein, J. F. Joanny, and J. Bibette, Phys. Rev. Lett. 94, 038301 (2005).

29. A. Koenig, P. Hébraud, C. Gosse, R. Dreyfus, J. Baudry, E. Bertrand, and J. Bibette, Phys. Rev. Lett. 95, 128301 (2005).

30. G. Taylor, Proc. R. Soc. A 209, 447 (1951).

31. M. Reichert, and H. Stark, Eur. Phys. J. E 17, 493-500 (2005).

32. O. Kratky and G. Porod, Recl. Trav. Chim. Pays-Bas 68, 1106 (1949).

33. J. R. Blake, Proc. Camb. Phil. Soc. 70, 303 (1971).

34. J. Happel and H. Brenner, Low Reynolds Number Hydrodynamics (Noordhoff, Leyden, 1973).

35. L. D. Landau and E. M. Lifschitz, Lehrbuch der Theoretischen Physik Band VII: Elastizitätstheorie (Akademie Verlag, Berlin, 1991)

36. J.K.G. Dhont, An Introduction to Dynamics of Colloids (Elsevier, Amsterdam, 1996).
37. In our modeling, we use a center-to-center distance $3 a$ of the particles in the filament (where $a$ is the particle radius). In this case, the Rotne-Prager approximation deviates from the full multipole results of Ref. 38 by at most $5 \%$. We checked this by using the program Hydrolib (see Ref. 39]).

38. B. Cichocki, B. U. Felderhof, K. Hinsen, E. Wajnryb, and J. Blawdziewicz, J. Chem. Phys. 100, 3780 (1994).

39. K. Hinsen, Comput. Phys. Commun. 88, 327 (1995).

40. H. A. Lorentz, Zittingsverlag Akad. v. Wet. 5, 168 (1896).

41. E. R. Dufresne, M. Todd, M. P. Brenner, and D. G. Grier, Phys. Rev. Lett. 85, 3317 (2000).

42. L. Durlofsky, J. F. Brady, and G. Bossis, J. Fluid Mech. 180, (1987).

43. G. Bossis, A. Meunier, and J. D. Sherwood, Phys. Fluids A 3, 1853 (1991).

44. J. W. Swan and J. F. Brady, Phys. Fluids 19, 113306 (2007).

45. S. Melle, O. G. Calderón, M. A. Rubio, and G. G. Fuller, Phys. Rev. E 68, 041503 (2003).

46. A. Cēbers and I. Javaitis, Physical Review E 69, 021404 (2004).

47. Another obvious way of achieving a non-reciprocal motion of the superparamagnetic filament would be to vary the magnetic field strength non-symmetrically during the actuation cycle. However, in our simulations we find that this method is less robust and effective.

48. J. Blake, J. Fluid Mech. 55, 1 (1972).

49. Note that formally the induced flow along the $y$ direction should also depend on the force components $F_{x i}$ and $F_{z i}$. Besides the fact that $F_{x i}=0$ in our case, by symmetry the

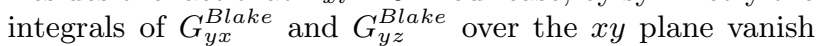
and additional contributions to Eq. (27) do not occur.

50. M. Fermigier, private communication.

51. G. Maret, private communication.

52. R. B. Jones, and R. Kutteh, Phys. Chem. Chem. Phys. 1, 2131 (1999). 